\title{
REPRÉSENTATIONS DU POLITIQUE DANS LES FILMS CONTEMPORAINS DÉDIÉS AUX ANNÉES 1970: LECTURES EN CREUX DU PRÉSENT
}

\begin{abstract}
Résumé: Le cinéma italien des années 2000 a vu se multiplier la production de films dédiés aux années 1970. L'analyse de la production cinématographique consacrée à cette décennie, dont la complexité est loin d'être suffisamment éclaircie, pousse à s'interroger non seulement sur les phénomènes historico-politiques les plus sollicités dans ces films - par exemple la focalisation de l'attention des cinéastes sur les facettes les plus dramatiques de cette période, comme la stratégie de la tension ou la lutte armée - mais aussi sur la façon dont ces thématiques sont souvent traitées : caractérisation de la figure d'Aldo Moro, représentation en huis clos du phénomène de la violence politique, rapport entre conflit public et drame privé, présence ou absence des revendications de la société civile.
\end{abstract}

Riassunto: Nel cinema italiano degli anni Zero si sono moltiplicati i film sugli anni Settanta. L'analisi della produzione cinematografica dedicata a questo periodo, la cui complessità non è ancora stata sufficientemente esaminata, conduce a interrogarsi non solo sui fenomeni storico-politici più rappresentati in tali film - ad esempio l'attenzione focalizzata sugli aspetti più drammatici del periodo considerato, come la strategia della tensione o la lotta armata - ma anche sul modo in cui queste tematiche sono spesso trattate: caratterizzazione della figura di Aldo Moro; rappresentazione prevalentemente a porte chiuse del fenomeno della violenza politica; rapporto tra conflitto pubblico e dramma privato; presenza o assenza di rivendicazioni da parte della società civile.

Mon propos est ici d'interroger, d'un point de vue civilisationnel, la construction de l'imaginaire lié à la représentation d'une période particulièrement sensible et complexe de l'histoire récente de l'Italie: les années soixante-dix du XX ${ }^{\mathrm{e}}$ siècle. Il ne s'agira donc pas de fournir une analyse filmique technique ni de formuler des jugements de valeur de type esthétique sur la qualité de l'œuvre du point de vue de sa réalisation (photographie, maestria des acteurs, montage, son), mais d'aborder ces aspects seulement dans la mesure où ils renseignent sur le regard que le cinéma porte sur les événements historiques traités. 
Une première observation concerne la question, largement débattue dans le domaine de la critique cinématographique, du rapport entre films de fiction et histoire ${ }^{1}$. À ce propos, Lino Micciché, en parlant des films de genere storico, rappelle qu'un film parle toujours du présent de sa réalisation, de l'époque dans laquelle il a été réalisé, même lorsqu'il s'agit d'un film dont la matière de la narration est historique: "In effetti, il soggetto, vivente oggi, esprime, da una parte e inevitabilmente, un punto di vista sul proprio presente; dall'altra parte, metaforizzando il passato, esprime anche un punto di vista sul passato $»^{2}$.

Dans ce sens, la production de films de fiction dédiés à la décennie soixante-dix à partir des années 2000, les narrations et les images qu'ils proposent sur cette période, sont intéressants en ceci qu'ils sont à relier avec l'actualité politique de l'époque à laquelle les films ont été conçus, et plus largement en ce qu'ils nous renseignent sur la construction d'un imaginaire social et politique. Qu'est-ce que ces films disent de l'époque sur laquelle ils se penchent et donc aussi sur l'époque présente? C'est à partir d'un tel questionnement que l'on pourra s'interroger sur la valeur politique des films qui aujourd'hui racontent les années 1970, et sur les rapports qu'ils tissent avec les deux pôles qui constituent le noyau de réflexion de ce colloque: film politique et film engagé.

Sur un arc temporel d'environ dix ans, de 2003 à 2014, plus d'une douzaine de films de fiction portant sur les années 1970, aussi bien pour le grand que le petit écran ${ }^{3}$, ont été réalisés ${ }^{4}$. À un niveau macro, il s'agira donc de questionner les faits et les phénomènes historico-politiques les plus sollicités par cette production filmique qui nous renseignent sur ce qui fascine ou pose problème dans la représentation et la compréhension de la décennie soixante-dix. À un niveau micro, il est possible de s'interroger sur l'image fournie des sujets choisis: caractérisation des personnages principaux, qu'ils soient protagonistes ou antagonistes, représentation des institutions et de la classe politique, de la société civile, des mouvements collectifs.

1. La bibliographie sur ce sujet étant très étendue, nous renvoyons à l'article d'A. O'Leary, «Cinema, storia, Italia. Appunti per una ricerca in espansione», Studi culturali, XII, n 2, août 2015, p. 1-19.

2. L. Micciché, "Alcune considerazioni teoriche su cinema e storia», La valle dell'Eden, VI, $\mathrm{n}^{\circ}$ 12-13, juillet-décembre 2004, Dossier Cinema e storia, G. Alonge, G. Carluccio, F. Villa (éd.), p. 20.

3. Concernant la télévision, voir l’article de V. Roghi, «Gli anni Settanta e la Tv degli ultimi dieci anni», Novecento.org [en ligne], n 2, 2014, DOI:10.12977/nov34, http://www.novecento. org/uso-pubblico-della-storia/vittime-gli-anni-settanta-e-la-tv-degli-ultimi-dieci-anni-533/.

4. A. O'Leary affirme que les films portant sur les «années de plomb» semblent désormais se constituer en genre ("Italian cinema and the "anni di piombo" ", Journal of European Studies, $\mathrm{n}^{\circ} 40$ (3), 2010, p. 243-257). 


\section{Narrations, dénominations, mémoire}

La plupart des films de fiction sur la décennie soixante-dix choisissent comme sujet principal de leur narration la lutte armée, les massacres et les mystères de cette période ${ }^{5}$ : à titre indicatif, on peut considérer que six portent sur le terrorisme de gauche et les groupes révolutionnaires ${ }^{6}$, six autres, sans en faire l'élément principal, lui consacrent une place importante $^{7}$ et au moins deux enfin portent sur les «massacres» accomplis par les pouvoirs politiques corrompus ${ }^{8}$.

L'on remarque d'emblée que la plupart de ces films semblent refléter le débat historiographique concernant la dénomination de cette période: en plaçant l'accent principalement sur la question de la violence politique, ils fournissent une vision des années 1970 conforme à l'usage courant de l'expression "années de plomb» qui repose sur une des interprétations possibles du titre du film de Margareth von Trotta, Die Bleierne Zeit ${ }^{9}$. De ce fait, ils semblent en revanche laisser de côté d'autres aspects essentiels de celle qui a été également appelée la «saison des mouvements» : libération de l'individu et expérience du collectif, avancées sociales. La question de la dénomination de la période que nous avons choisie comme champ de recherche est complexe, en ceci qu'elle induit une lecture idéologique. Si l'expression «années de plomb» s'est imposée dans l'usage courant ${ }^{10}$,

5. Seulement deux films semblent constituer une exception: Lavorare con lentezza (2004) de Guido Chiesa sur l'expérience de Radio Alice à Bologne et La signorina Effe (2008) de Wilma Labate sur la Marche des 40 ooo le 14 octobre 1980 et la défaite des luttes ouvrières à la Fiat.

6. Buongiorno, notte (2003) de Marco Bellocchio; Piazza delle cinque lune (2003) de Renzo Martinelli; Se sarà luce sarà bellissimo. Moro un'altra storia (2008) d'Aurelio Grimaldi; Aldo Moro il presidente (2008) de Gian Luca Maria Tavarelli; La prima linea (2009) de Renato de Maria; Sangue Sparso (2014) d'Emma Moriconi.

7. La meglio gioventù (2003) de Marco Tullio Giordana; Arrivederci amore, ciao de Michele Soavi (2006); Guido che sfidò le Brigate Rosse (2007) de Giuseppe Ferrara; Mio fratello è figlio unico (2007) de Daniele Luchetti; Il sorteggio (2010) de Giacomo Campiotti; Gli anni spezzati (2014) de Graziano Diana.

8. Romanzo criminale (2005) de Michele Placido; Romanzo di una strage (2012) de Marco Tullio Giordana.

9. Comme le rappelle Giovanni De Luna (dans les notes de son chapitre «Tra Memoria e oblio", in Le ragioni di un decennio: 1969-1979. Militanza, violenza, sconfitta, memoria, Milan, Feltrinelli, 2008), l'expression trouve son origine dans l'interprétation italienne du titre du film de Margareth von Trotta, Die Bleierne Zeit, qui avait été présenté au festival de Venise en 1981 avec deux titres, Tempi di piombo et Gli anni plumbei, l'adjectif «bleiern» pouvant se traduire par «oppressant». Ce n'est qu'à la sortie en salle que le titre italien est devenu Anni di piombo.

10. Voir l'article de C. Lettieri, «L'Italie et ses Années de plomb. Usages sociaux et significations politiques d'une dénomination temporelle», Mots. Les langages du politique [en ligne], $\mathrm{n}^{\circ} 87,2008$, DOI : 10.4000/mots.12032, http://mots.revues.org/12032. 
il est important de souligner que cette appellation met l'accent sur les conflits armés et sur le terrorisme, qui furent certes un des phénomènes les plus marquants de la période, mais auxquels il est impossible de réduire le foisonnement et la complexité des mouvements politiques et sociaux qui ont animé la décennie ${ }^{11}$. C'est pourquoi nous préférons parler des "années 1970 » ou utiliser l'expression "saison des mouvements», qui, selon la définition de Marco Grispigni, indique cette phase historique "nella quale una serie di movimenti sociali (diversi fra loro, ma con alcune caratteristiche unificanti) furono tra i protagonisti principali della scena politica, sociale e culturale» ${ }^{12}$.

À l'intérieur de cette production cinématographique, il est inévitable de constater le grand nombre de films dédiés à l'affaire Moro, donnant lieu à un «Todo Moro», pour reprendre le jeu de mots que Christian Uva a créé à partir du titre d'un roman de Sciascia ${ }^{13}$. Que l'on pense au presque «instant movie» de Giuseppe Ferrara, Il caso Moro (1986) réalisé à moins de dix ans de distance des faits, aux déjà cités Buongiorno, notte, Piazza delle cinque lune, Se sarà luce sarà bellissimo. Moro un'altra storia, pour finir avec Aldo Moro il Presidente.

Ainsi que l'écrit l'historien spécialiste de la décennie soixante-dix, Guido Panvini,

«Piazza Fontana»e il «caso Moro» sono diventati due topoi in grado di assorbire in sé la complessità della storia italiana degli anni Settanta e di condizionarne la narrazione, come se vi fosse stata una catena di eventi predeterminata e destinata a generarsi, evolversi e compiersi nel lasso temporale compreso tra il 12 dicembre del 1969 e il 9 maggio del $1978^{14}$.

De plus, la représentation fournie par certains de ces films, en reproduisant la logique même du mystère et de la théorie du complot sans qu'il y ait un responsable clairement identifiable - l'historien cite par exemple le cas

11. A. O'Leary rappelle d'ailleurs que Margareth von Trotta voulait par son titre souligner le poids de l'histoire, alors que l'association entre plomb et projectiles n'évoque finalement que la violence politique d'extrême gauche en occultant le stragismo d'extrême droite. Cf. A. O'Leary, "Italian cinema and the "anni di piombo" ", p. 244.

12. M. Grispigni, Elogio dell'estremismo: storiografia e movimenti, Rome, Manifestolibri, 2000, p. 21. L'expression «stagione dei movimenti » a été utilisée pour la première fois dans l'ouvrage collectif Il sessantotto. La stagione dei movimenti (1960-1979), Rome, Edizioni associate, 1988.

13. C. Uva, Schermi di piombo. Il terrorismo nel cinema italiano, Soveria Mannelli, Rubbettino, 2007, p. 69.

14. G. Panvini, «La sfida al labirinto. Narrazione cinematografica e interpretazione storica di fronte al "mistero" della violenza», in Cinema e misteri d'Italia, C. Uva (éd.), Soveria Mannelli, Rubbettino, 2011, p. 189. 
de Romanzo criminale de Michele Placido - risque de laisser le spectateur politiquement démuni, impuissant comme face à une calamité naturelle ${ }^{15}$. Guido Panvini voit en cela un problème lié directement au fait que la narration de ces années-là est influencée par la «mémoire», une mémoire qui, au lieu d'éclaircir la vision de cette période, jette un voile d'opacité sur les événements et se fait expression de l'incapacité, voire, dans certains cas, de la «non volontà di raccontare quanto successo realmente in quegli anni» ${ }^{16}$.

Cette mémoire, rappelle-t-il, est souvent à l'origine des narrations filmiques (comme dans le cas des mémoires de Anna Laura Braghetti pour Buongiorno, notte ou de Sergio Segio pour La prima linea de Renato de Maria ${ }^{17}$ ), mais elle peut intervenir aussi, pourrions-nous ajouter, en tant que «mémoire» des réalisateurs eux-mêmes, qui ont été impliqués plus ou moins directement dans l'histoire qu' ils sont en train de raconter: on pense à Michele Placido qui met en scène sa jeunesse partagée entre sa vocation théâtrale et son travail dans la police dans Il grande sogno (2009), ou au militantisme de Bellocchio dans un groupe marxiste-léniniste.

\section{Un huis clos politique et psychologique}

Concernant la représentation de la lutte armée, on est frappé dans nombre de films ${ }^{18}$ par la dimension de «huis clos » politique et psychologique qui fait apparaitre le recours à la violence comme séparée de la réalité sociale et politique des luttes collectives.

On pense à une des scènes emblématiques du film La meglio gioventù dans laquelle Nicola Carati (Luigi Lo Cascio) entre dans la chambre enfumée de son appartement où sa compagne Giulia Monfalco (Sonia Bergamasco), qui choisira par la suite la lutte armée, est enfermée avec d'autres militants

15. «Cosi la rappresentazione filmica della strage di Bologna del 2 agosto 1980 (si pensi ad esempio a Romanzo criminale di Michele Placido, 2005), insistendo sul "carattere apparentemente senza senso del massacro", spinge lo spettatore a pensare all'attentato come a una calamità naturale» (ibid., p. 195).

16. Ibid., p. 191: "Spesso ci si dimentica, però, che la memoria, oltre a essere un vettore di conoscenza, è anche uno strumento di lotta politica. Omissioni, recriminazioni, contraddizioni, omertà e falsità abbondano nella memorialistica de-gli ex appartenenti ai gruppi armati di sinistra [...] in uno strano parallelismo con le memorie di molti esponenti della Democrazia Cristiana, come, ad esempio, quelle di Paolo Emilio Taviani, Giulio Andreotti, Francesco Cossiga, quando nel racconto ci si avvicina ai momenti più drammatici di quella stagione. All'opacità dei poteri, parafrasando Foucault, si è aggiunta quella del "contropotere". Trasmessa dalla memorialistica, tale opacità si è poi estesa alla narrazione cinematografica».

17. A.L. Braghetti, P. Tavella, Il prigioniero, Milan, Mondadori, 1998; S. Segio, Miccia corta. Una storia di prima linea, Rome, Derive Approdi, 2005.

18. Je citerai ici surtout des séquences tirées de Buongiorno, notte et de La meglio gioventù à cause du caractère exemplaire de ces films et de leur grand succès auprès du public. 
en laissant la petite Sara abandonnée à elle-même dans la pièce d'à côté, ce que le personnage commente de la sorte: «i posti chiusi fanno male alle bambine e anche al cervello della gente».

Cette sensation de huis clos est particulièrement évidente dans le cas de Buongiorno, notte qui, dans l'intention du réalisateur, veut donner à voir l'enlèvement de Moro de l'intérieur de la cellule terroriste, voire même de la conscience de l'une des brigadistes, le personnage de Chiara (Maya Sansa). C'est pourquoi Buongiorno, notte se présente comme un holekeyfilm, ainsi que le rappelle Christian Uva, un film où le regard du protagoniste, et donc celui du spectateur, sont très souvent filtrés par un judas, et ce, notamment, lorsqu'il s'agit de donner à voir Aldo Moro ${ }^{19}$. Dans ce film dominent les espaces fermés et sombres (l'appartement censé représenter la «prison du peuple», le lieu de travail de Chiara) auxquels s'opposent quelques rares séquences filmées à l'extérieur ${ }^{20}$. Il s'agit d'ailleurs de scènes dont la valeur symbolique est forte: la recherche des canaris envolés dans le jardin de la "prison du peuple», le déjeuner avec l'oncle de Chiara et ses amis anciens résistants, et bien évidemment la fin du film avec la promenade de Moro dans une Rome matinale que Bellocchio fait rêver par la protagoniste.

Qu'il s'agisse de stratégies narratives visant à rendre compte de la durée de la captivité du statista démocrate chrétien, ses cinquante-quatre jours de prigionia, ou alors de la crise, au sens étymologique du terme, de la séparation, que l'enlèvement de Moro (ou plutôt sa défaite, culminant dans son assassinat) va produire au sein du movimento et au sein de la conscience même de certains extrémistes, on peut néanmoins s'interroger sur la rareté des représentations des rapports qui avaient existé entre les Brigades rouges et les groupes de la gauche extra-parlementaire ${ }^{21}$. Car s'il est vrai que les militants de gauche et d'extrême gauche avaient commencé à exprimer leur distance par rapport à l'usage de plus en plus diffus de la violence en concomitance avec l'enlèvement de Moro (on pense au slogan

19. C. Uva, «Tableaux mourants: l'immagine di Aldo Moro tra fotografia, pagina scritta e cinema», The italianist, $\mathrm{n}^{\circ} 31,2011$, p. 258-269.

20. Ces séquences frappent d'autant plus que les autres images de lieux extérieurs qui apparaissent dans le film sont de fait enfermées dans l'écran de la télévision.

21. Dans Il caso Moro de Giuseppe Ferrara, cette question était en revanche évoquée à plusieurs reprises, en faisant néanmoins apparaître la classe ouvrière et le mouvement étudiant unanimement opposés aux brigadistes et trahissant de la sorte le positionnement idéologique du metteur en scène. On pense, par exemple, à l'utilisation des images d'archives du discours du syndicaliste Luciano Lama qui avait assimilé les terroristes à de simples criminels, ou à la rencontre entre deux terroristes et un représentant du movimento qui leur dit: «Ma lo volete uccidere veramente? Guardate che nel movimento non c'è un cane che è d'accordo con voi». 
de Lotta Continua «né con lo Stato né con le BR»), la possibilité d'un recours à la violence révolutionnaire informe nombre des luttes de la décennie.

D'ailleurs, cette dimension de huis clos s'accompagne d'une représentation des terroristes en aliénés, par la mise en exergue d'une déviance psychologique pathologique, comme lorsque Bellocchio fait répéter à ses terroristes comme un mantra la phrase: «la classe operaia deve dirigere tutto, la classe operaia deve dirigere tutto".

Ceci va de pair souvent avec la mise en scène des terroristes femmes ${ }^{22}$, ce que le chercheur Alan O'Leary lit notamment comme une mise en image de la crise du patriarcat dans ces années. Toutefois, plutôt que l'expression de l'importance du mouvement féministe, il nous semble en réalité que nous retrouvons là le cliché de la femme hystérique, dont la violence aurait à voir avec un rapport problématique à son utérus en tant qu'organe de la reproduction: d'où le choix dévié de la violence au lieu du choix «naturel» de la procréation.

Là encore, il est possible de citer en exemple le personnage de Chiara dans Buongiorno, notte: son évidente absence d'instinct maternel lorsqu'une voisine lui confie son bébé, sa fragilité psychologique. Ou encore le personnage de la terroriste Giulia dans La meglio gioventù qui apparaît, au fur et à mesure que la saga se déroule, comme une névrosée, mère insensible qui abandonne sa fille. Cette représentation des terroristes en déviants semble correspondre à un processus de dépolitisation de leur discours et de leurs choix. D'ailleurs, comme l'affirme Alan O'Leary, «le scelte di Giulia non sono mai investigate a fondo, e il suo passaggio alla lotta armata sembra saltare tutta una fase intermedia di attività politica che sarebbe potuta essere tipica della sinistra extraparlamentare agli inizi degli anni ${ }^{\prime} 70 »^{23}$.

Il n'est évidemment pas dans notre intention de légitimer ni de défendre le recours à la violence de la part des Brigades rouges ou de la part d'autres groupes armés agissant à l'époque. Il nous semble toutefois que les films cités, comme d'ailleurs un certain nombre d'autres pellicules - Aldo Moro il Presidente de Tavarelli, La prima linea de Renato de Maria, sans parler d'Arrivederci amore ciao de Michele Soavi - n'arrivent pas, ou ne cherchent pas à faire comprendre l'origine politique du choix du passage à la lutte armée de la part d'un certain nombre de militants d'extrême gauche, ni leur décision d'une intensification du conflit contre

22. Voir aussi R. Glynn, «Terrorism, a Female Malady?», in Terrorism Italian Style: the Representation of Terrorism and Political Violence in Contemporary Italian Cinema, R. Glynn, G. Lombardi, A. O'Leary (dir.), Londres, IGRS Books, 2012, p. 117-132.

23. A. O'Leary, Tragedia all'italiana. Cinema e terrorismo tra Moro e memoria, Tissi, Angelica editore, 2007, p. 195. 
l'État. Alors que, comme le rappelle la politologue Isabelle Sommier, la radicalisation de la nouvelle gauche et le recours à la violence révolutionnaire ne peuvent être compris en dehors de «l'environnement politique propre à chaque pays", et donc, en ce qui concerne le cas italien, en dehors de la stratégie de la tension, des mesures répressives de la part de l'État et du «compromis historique»: «Ce n'est pas une coïncidence si la période de la théorisation du compromis historique (1973-1975) est aussi celle du basculement progressif des premiers groupes clandestins vers la lutte armée, qui se déploie entre les deux échéances électorales de 1976 et 1979 et culmine avec l'affaire Moro $»^{24}$.

\section{Le rapport public-privé:}

\section{la réduction du conflit politique à un drame familial?}

Nombreux sont les films qui placent au cœur de leur narration la représentation du noyau familial et des conflits et des crises qui le traversent en parallèle avec les conflits ou crises politiques. L'on pense bien évidemment à la saga familiale racontée dans La meglio gioventù, à l'opposition entre les deux frères dans Mio fratello è figlio unico de Daniele Lucchetti, à la crise familiale dans Il grande sogno de Michele Placido (film qui se termine avec la figure du père mourant rassemblant autour de son chevet ses enfants comme des brebis égarées), aux représentations des deux familles dans Romanzo di una strage: le commissaire Calabresi avec sa femme enceinte, puis avec ses deux enfants en bas âge, et l'anarchiste Pinelli avec ses deux filles et sa femme.

Mais cette dimension familiale apparaît également dans le portrait de Moro en pater familias ${ }^{25}$ qui revient dans nombre de pellicules, parfois directement par la représentation de la famille du chef démocrate chrétien, sa femme, ses enfants et son petit-fils Luca (c'était le cas dans le film de Ferrara mais aussi dans celui de Tavarelli), très souvent indirectement, au travers du leitmotiv de la lecture de la dernière lettre de Moro à sa femme ${ }^{26}$.

Ceci relève certainement de plusieurs facteurs, aussi bien d'ordre dramaturgique qu'historiographique. D'un côté, la dimension privée, personnelle, familiale des histoires racontées, motif typique du cinéma italien, permet

24. I. Sommier, La violence révolutionnaire, Paris, Presses de Sciences Po, 2008, p. 106-107.

25. "Non a caso, protagonista di Buongiorno, notte è una "sacra famiglia" (è il titolo del testo di Marx ed Engels che la brigatista legge prima di addormentarsi) in cui la vecchia, canonica tematica di Bellocchio e della sua generazione, ovvero la messa a morte dei Padri, trova la sua definitiva e forse ultima celebrazione» (C. Uva, "Tableaux mourants: l'immagine di Aldo Moro tra fotografia, pagina scritta e cinema», p. 263).

26. Par exemple dans les films de Ferrara, Bellocchio, Tavarelli et Grimaldi. 
non seulement une plus grande caractérisation des personnages mais aussi la possibilité d'une empathie avec le spectateur, dans le but peut-être de rendre l'Histoire plus vivante, plus proche de nous.

D'un autre côté, on peut y voir peut-être la tentative de rendre compte de l'imbrication effective qui s'était créée dans nombre de trajectoires existentielles entre ces deux pans de la vie d'un individu. En effet, non seulement les mouvements nés avec Mai 68 ont été lus comme le résultat d'un affrontement générationnel, une révolte des fils contre leurs pères, réels ou symboliques, mais la décennie soixante-dix a été également celle où l'on est passé du «tutto politico» à "il personale è politico» comme le récitaient des slogans de l'époque. En ce sens, ainsi que l'écrit Alan O’Leary, le trope de la famille comme image de la nation, qui est une constante de la représentation cinématographique des années 1970, pourrait en effet évoquer aussi bien le conflit œdipien entre fils et père que l'idée d'une guerre civile lorsque ce sont deux frères qui s'affrontent ${ }^{27}$.

Toutefois, cette centralité de la représentation de la famille, en jouant souvent sur les cordes du pathos et du sentimentalisme, tend à réduire le conflit politique à un drame familial, et de fait semble ramener le public au privé. De ce point de vue, par exemple, il serait intéressant d'analyser les choix formels caractérisant la représentation des figures des victimes, et de Moro comme symbole de toutes les victimes, qui, en répondant à une volonté légitime de reconnaissance de ces parcours existentiels brisés, insistent sur les drames personnels et familiaux ${ }^{28}$. Comme l'écrit Vanessa Roghi, "se l'ideologia sorregge le memorie degli ex brigatisti, niente può spiegare, in una dimensione privata, perché un padre, un marito, un fratello abbiano perso la vita ${ }^{29}$.

La politique ne serait rien d'autre que ce qui vient briser la paix sociale, certes, mais avant tout familiale. Et on glisserait en quelque sorte de «il personale è politico» à «il politico è personale». Ce glissement s'opère au profit d'un dévidement idéologique qui rend les conflits de ces années-là encore plus insensés qu'ils n'ont pu l'être. De ce fait, il n'est peut-être pas si surprenant qu'aussi bien Bellocchio que Giordana, par exemple, prennent leurs distances par rapport à un discours politique qui est désormais connoté

27. A. O'Leary, «Terrorism and the anni di piombo in Italian cinema», ISLG Bulletin, $\mathrm{n}^{\circ}$ 9, 2010, p. 40-48.

28. Uva parle du «divismo» qui finit par entourer la figure de Moro, «iconografia del "martire per eccellenza" degli anni di piombo, ciò che efficacemente Alan O'Leary ha definito "la sineddoche" di tutte le morti di quella stagione» ("Tableaux mourants: l'immagine di Aldo Moro tra fotografia, pagina scritta e cinema», p. 258-259).

29. V. Roghi, «Gli anni Settanta e la Tv degli ultimi dieci anni». 
négativement au profit d'une valorisation du «privé», du "particulier», $\mathrm{du}$ «personnel ${ }^{30}$.

Ce processus, comme il a été suggéré aussi par d'autres, pourrait correspondre à une évolution même de la gauche italienne et plus largement de la place de la politique dans nos sociétés ${ }^{31}$. Comme l'affirme le cinéaste Guido Chiesa, au sujet de La meglio gioventù, il s'agirait peut-être de répondre à la «necessità di riscrivere la storia secondo le traiettorie ideologiche dei partiti della sinistra ${ }^{32}$, lesquels, dans l'objectif d'acquérir une légitimité en tant que partis au gouvernement, souhaitent prendre leur distance par rapport à leur origine révolutionnaire ${ }^{33}$ et substituer à l'idée d'une libération des masses prolétariennes une perspective de salut individuel ${ }^{34}$.

\section{Pour une contre-histoire des années 1970}

Se sarà luce sarà bellissimo - Moro: un'altra storia d'Aurelio Grimaldi a été défini le plus «politiquement incorrect» des longs métrages consacrés à la destinée du statista democrate chrétien. D’ailleurs, comme l'a précisé le metteur en scène lui-même, ce film n'est pas tant dédié à l'affaire Moro

30. Voir par exemple les interviews réalisées par M. Fantoni Minnella dans Non riconciliati. Politica e società nel cinema italiano dal neorealismo a oggi, Turin, UTET, 2004. Marco Bellocchio: «Mentre in passato si diceva "cinema politico" perché tutto era politico, il privato era politico, adesso nella coscienza e nell'inconscio delle persone la politica è qualcosa di indistinto, di poco chiaro, a volte di fortemente negativo, e di conseguenza parlare di cinema politico è una definizione smarrita [...]. Il mio film Buongiorno, notte non è politico, parla di politica, però è un film privatissimo» (p. 223). Marco Tullio Giordana: «Non mi considero, non mi sono mai considerato, un esponente del cosiddetto cinema politico. [...] La meglio gioventù, un film che bypassa completamente la politica, che si occupa d'altro e quell'altro oltretutto è proprio la ragione della sua riuscita, del suo grande successo anche internazionale. Quelli che i sondaggi chiamano "la gente" non ne possono più di veder inquinato ogni discorso dalla "politica", di sentirne parlare, di veder fissata l'agenda delle proprie emozioni, della propria storia personale solo attraverso il filtro vagamente minaccioso del politically correct » (p. 280-283).

31. Voir par exemple ce qu'écrit A. O’Leary sur La meglio gioventù: «È una celebrazione forgiata secondo la modalità postmoderna dell'impegno esemplificata da un "ritrarsi dell'impegno da ideologie macropolitiche, sinistra / destra, a favore di iniziative micropolitiche, basate sulla comunità" "(Tragedia allitaliana. Cinema e terrorismo tra Moro e memoria, p. 194).

32. "Cineasti a confronto. Guido Chiesa, Wilma Labate, Francesco Patierno, Marco Turco", in C. Uva, Schermi di piombo. Il terrorismo nel cinema italiano, p. 247-248.

33. Cf. A. O'Leary: «La cifra sentimentalistica è funzionale alla negazione del progetto di cancellazione di un patrimonio di violenza, un progetto necessario per trasformare la sinistra in un centrosinistra con legittime velleità di potere (anziché un'opposizione con un ruolo semplicemente morale) nella Seconda Repubblica» (Tragedia allitaliana. Cinema e terrorismo tra Moro e memoria, p. 198).

34. Voir M. Fantoni Minnella, Non riconciliati. Politica e società nel cinema italiano dal neorealismo a oggi, p. 126-127. 
qu'au phénomène du terrorisme dans son ensemble, et prend la forme d'une «radiographie de l'Italie» des années $1970^{35}$, d'une contre-histoire de la période, comme le titre le laisse entendre.

Le film aurait dû être à l'origine une trilogie comprenant une première partie sur la captivité de Moro, une deuxième sur l'expérience de la prison par les brigadistes et par d'autres militants d'extrême gauche au début des années 1980, et une troisième consacrée à des épisodes parallèles à la captivité de Moro. La faillite du producteur a de fait empêché la finalisation de la trilogie, dont $80 \%$ des séquences étaient déjà filmées. Ce n'est que quatre ans après le tournage que Grimaldi décidera enfin de fusionner la première et la troisième partie de la trilogie en un seul film, avec des moyens financiers très limités qui expliquent la faible qualité de l'image et du son, qui sera distribué en 2008 uniquement en DVD.

Grimaldi met en regard l'emprisonnement du chef de la Démocratie chrétienne avec d'autres épisodes d'incarcération, liés ceux-là à la violence étatique: l'arrestation d'une enseignante, interprétée par l'actrice Lalla Esposito, enfermée pour avoir durement critiqué l'État dans son établissement scolaire, et celle d'un jeune d'extrême gauche, interprété par Fabrizio Raggi, que l'on accuse sans fondement d'être un terroriste.

Tout le film est construit sur ces histoires menées en parallèle. Les scènes de l'incarcération de Moro (Roshan Seth) dans la "prison du peuple» et son interrogatoire, tendu mais très respectueux de sa personne, alternent donc avec les scènes, d'un côté, de l'arrestation de la jeune professeur par le commissaire Achille Crollo (Gaetano Amato), sa détention dans une cellule du commissariat sans possibilité de boire ni de se laver pendant plusieurs jours, les interrogatoires violents qu'elle doit subir et sa suspension de l'enseignement. De l'autre côté, le spectateur assiste aux scènes des sévices infligés au jeune militant auquel on veut faire avouer par la torture sa prétendue appartenance aux Brigades rouges.

Cette alternance, due en partie aux aléas de production, est l'une des caractéristiques qui conduisent à une lecture plus articulée de la période examinée. Elle trouve son apogée au moment où la voix de Moro, lisant la lettre qu'il avait adressée au ministre de l'Intérieur Francesco Cossiga, se superpose aux images de la torture du jeune militant:

Caro Francesco, prescindo volutamente da ogni aspetto emotivo e mi attengo ai fatti. Mi trovo ristretto sotto un dominio pieno e incontrollato e sottoposto come presidente del nostro partito a un processo diretto a accertare le nostre responsabilità. Per la prima volta i miei carcerieri mi hanno mostrato un

35. Interview d'Aurelio Grimaldi par Miriam Tola, http://news.cinecitta.com/IT/it-it/ news/54/61709/aurelio-grimaldi.aspx, 14 octobre 2004. 
quotidiano che mi hanno però vietato di citare. La lettura di quel giornale mi ha deciso. Qui c'è in gioco una vita umana e la ragion di Stato nasce e finisce col rispetto della vita umana. Ti imploro in ginocchio di non permettere più tutto questo e di considerare seriamente la possibilità di uno scambio di prigionieri. Che Iddio ti illumini e ti dia la forza di illuminare anche i nostri compagni di partito. Tuo Aldo Moro.

La supplication de Moro pour la défense de sa personne qui, par le biais du montage, semble en quelque sorte commenter les images de la torture infligée au jeune militant, se transforme ainsi en dénonciation de la violence étatique et des responsabilités politiques de la mort du statista comme des autres victimes de la période, y compris les militants des groupes extra-parlementaires.

Par ailleurs, dans cette tentative de fournir une contre-histoire de la période, Grimaldi choisit de s'écarter de la tendance à représenter Moro de façon apologétique et tend à privilégier une représentation de celui-ci en demi-teinte (du point de vue de l'image ceci est rendu par le clair-obscur qui accompagne souvent les apparitions du personnage). Si la situation de sa captivité le rend humainement touchant à cause de la fragilité, de la peur et de l'attachement à sa famille dont il fait preuve, le cinéaste essaie de montrer également son ambiguïté politique. Ceci est particulièrement évident lors de ses interrogatoires : son incapacité à se justifier, et le silence qui en découle au cours du premier interrogatoire, lorsque les Brigades rouges l'accusent d'avoir tenté de cacher son appartenance aux jeunesses fascistes (GUF), son attitude à l'égard des dictatures fascistes sud-américaines lorsqu'il était ministre de l'Intérieur et sa connaissance de la corruption de larges pans de son parti.

De plus, le long métrage de Grimaldi, en allant à contre-courant par rapport à la représentation en huis clos du phénomène de la lutte armée évoquée précédemment, souhaite au contraire montrer les liens entre les choix opérés par les Brigades rouges et le contexte politique. C'est pourquoi il donne à voir les débats internes à la gauche provoqués aussi bien par le «compromis historique » que par l'enlèvement de Moro. Ainsi certaines séquences montrent-elles l'expulsion de syndicalistes de la CGIL pour avoir pris position contre la stigmatisation des Brigades rouges de la part de Luciano Lama, tandis que d'autres relatent les discussions au sein d'une section romaine du PCI sur l'origine de gauche de la lutte armée et sur l'abandon d'une ligne politique révolutionnaire de la part du Parti communiste.

Grimaldi choisit enfin de placer son film au croisement entre fiction historique et témoignage, à travers les prises de paroles, externes au temps du récit, des familles des victimes de la violence politique des années 1970. 
Ces témoignages et la précédente évocation de la mort des deux jeunes militants ${ }^{36}$, allocutions extradiégétiques qui s'adressent directement au spectateur, veulent poser la question du lien entre l'histoire et le temps présent, entre cinéma et mémoire. Leur mesure, leur retenue (qui ne les rend pas moins touchants d'humanité) en opposition ouverte avec le pathos de certaines représentations de la mort des victimes, la recherche d'un équilibre entre le discours privé et le discours public ${ }^{37}$, et surtout la mise en cause d'une approche mémorielle visant à figer le passé sans tenter de le comprendre, se font l'expression d'une conception d'un cinéma militant, à la fois politique et engagé, qui veut agir dans le présent.

Ada Tosatti

Université Sorbonne Nouvelle - Paris 3

36. On retrouve, concernant ces témoignages, la recherche d'un équilibre entre les victimes de gauche et de droite. Au premier tiers du film, des acteurs interprètent Fausto Tinelli et Iaio Iannucci, connus à l'époque comme Fausto et Iaio, qui racontent leur assassinat le 18 mars 1978 à Milan par des extrémistes de droite. À la fin du film, ce sont encore des acteurs qui interprètent des membres de la famille de victimes des extrémistes de gauche: Luca, le petit-fils d'Aldo Moro, Alessandro, le fils d'un membre de son escorte, et Erminia Gemmato, la veuve d'une autre victime du terrorisme.

37. Le cinéaste fait dire, de façon provocatrice, à l'acteur qui incarne Luca Moro: «Che importanza può avere per tutti gli altri se sono malinconico oppure no, tanti altri sono chiusi e malinconici senza aver avuto il nonno che si chiamava Aldo Moro». 